\title{
Pericardial disease-anatomy and function
}

\author{
C R PEEBLES, MRCP, FRCR, J S SHAMBROOK, MRCP, FRCR and S P HARDEN, FRCS, FRCR
}

\begin{abstract}
Department of Cardiothoracic Radiology, Southampton General Hospital, Southampton University Hospitals NHS Trust, Southampton, UK
\end{abstract}

\begin{abstract}
Imaging of patients with suspected or known pericardial disease remains challenging. Echocardiography is the first-line investigation for pericardial disease but it has specific limitations in terms of its abilities to visualise the pericardium fully and to identify extracardiac pathology. Cardiac cross-sectional imaging by both MRI and CT has developed significantly and now has an important role in the investigation of pericardial disease. This article examines the appearances of both healthy and diseased pericardium using CT and MRI. The typical imaging findings across a wide range of conditions are illustrated and the roles of CT and MRI are reviewed. The relative merits and weaknesses of each modality are explored and the specific functional techniques that are available are introduced.
\end{abstract}

Received 22 July 2010

Revised 16 November 2010

Accepted 5 December 2010

DOI: $10.1259 / \mathrm{bjr} / 16168253$

(C) 2011 The British Institute of Radiology
The diagnosis of pericardial disease remains challenging. Symptoms are often insidious in onset and nonspecific, while clinical signs may be either difficult to elicit or absent. In addition, the initial investigations of electrocardiography (ECG), chest radiography and echocardiography lack sensitivity for identifying pericardial pathology [1-3]. Improved cross-sectional imaging techniques with CT and MRI now allow a detailed assessment of pericardial disease and its functional consequences. All radiologists who perform chest imaging need to be familiar with the appearances of both the normal pericardium and common pathologies. Knowledge of the relative merits of CT and MRI in different pericardial conditions is also important. This article reviews and illustrates the role of cross-sectional imaging in diagnosis and functional assessment across the spectrum of pericardial pathology. The normal appearances of the pericardium on CT and MRI are described, as are those of important congenital abnormalities. We also provide an introduction to the specific MRI and CT techniques available for the assessment of the pericardium, followed by a review of common pericardial pathologies and their imaging features.

\section{Imaging techniques for assessing pericardial disease}

Echocardiography is the first-line investigation for assessing possible pericardial disease. It is widely and readily available and is a quick and simple test. This examination is, however, limited in its ability to assess the pericardium because of a limited acoustic window, a limited field of view and its inability to image the entire pericardium [4]. As a result, cross-sectional imaging techniques are now widely accepted as the imaging modalities of choice for a comprehensive and accurate

Address correspondence to: Dr Charles Peebles, Department of Cardiothoracic Radiology, Southampton General Hospital, Tremona Road, Southampton SO16 6YD, UK. E-mail: Charles.peebles@ suht.swest.nhs.uk assessment of the pericardium. CT and cardiac magnetic resonance $(\mathrm{CMR})$ can both provide three-dimensional images of the heart and pericardium in any anatomical plane and can provide both structural and functional information. They are also less operator dependent than echocardiography and provide greater soft-tissue contrast.

\section{$C T$}

CT is a widely available technique that can be performed with or without ECG gating. The advantage of ECG-gated $\mathrm{CT}$ is the lack of cardiac motion on the images; thus, clear and motion-free images of the pericardium can be obtained. In general, however, CT scans are acquired without ECG gating as the majority of patients are scanned as part of the investigation of unexplained chest pain or shortness of breath, and hence a pericardial pathology may not be suspected initially. Even if pericardial pathology is being considered prior to $\mathrm{CT}$, reasonable images of the pericardium are usually obtained without ECG gating, so it is not an absolute requirement.

In general, CT is performed following the administration of intravenous (iv) contrast, and this allows an assessment of the size and morphology of the cardiac chambers. The thickness of the pericardium is readily measured, with the upper limit of normal being $2 \mathrm{~mm}$. Enhancement of thickened pericardium can also be determined, and this is useful in cases of suspected pericarditis or tumour infiltration. CT is particularly sensitive for identifying pericardial calcification, which is associated with pericardial constriction [5]. The density of pericardial effusions can be determined, with simple effusions having uniform low attenuation (with a Hounsfield unit of 0 ), whereas complex effusions, e.g. those resulting from blood or infection, are of increased density [6] (Figure 1).

CT has several advantages as an imaging modality: it has a short acquisition time of a few seconds, compared with up to $1 \mathrm{~h}$ for a CMR examination. CT makes a reliable assessment of pericardial calcification, whereas the appearance of calcification on CMR is variable and unreliable. The 


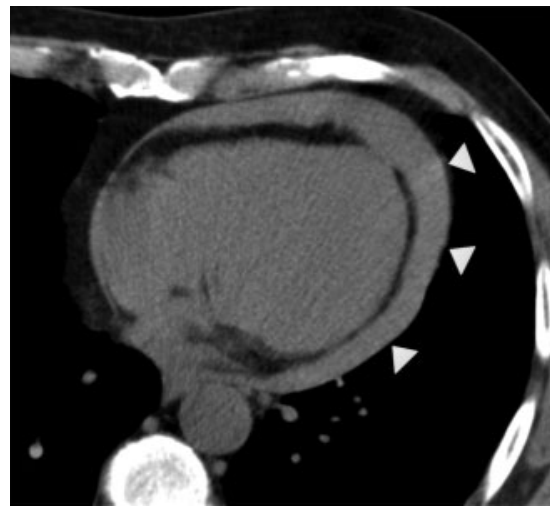

(a)

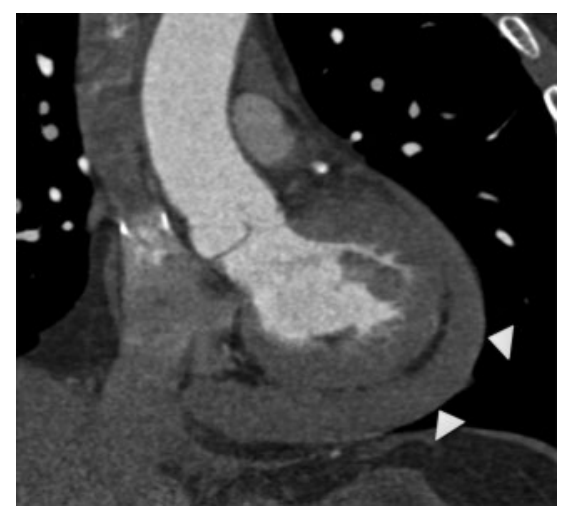

(b)

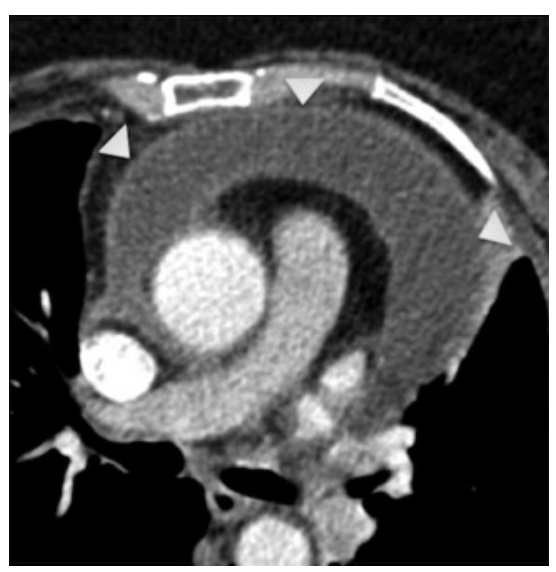

(c)

Figure 1. (a) Unenhanced CT scan demonstrating a haemorrhagic pericardial effusion (arrowheads); the effusion has an attenuation similar to that of muscle. (b) Coronal image in the same patient following contrast; the haemorrhagic nature of the effusion is more difficult to appreciate. (c) Axial-enhanced CT of a simple low-attenuation effusion (arrowheads).

principal disadvantage of CT is the lack of information on ventricular function, which is routinely provided by CMR. Secondary functional information, such as enlargement of the atria and venae cavae in cases of pericardial constriction, can be obtained by $\mathrm{CT}$, but ventricular functional parameters can be acquired only with retrospective ECGgated acquisitions, which are associated with an increased radiation dose. Most centres that use CT routinely would use CT to provide structural information and an echocardiogram to assess ventricular function.

\section{$C M R$}

The standard CMR protocol for investigating pericardial disease involves sequences to assess pericardial structure, ventricular function, flow and tissue characterisation. The basic sequences and their applications are set out below.

\section{Spin-echo black-blood imaging}

$T_{1}$ weighted images can accurately determine the thickness of the pericardium [7]. These images are often of higher quality when a saturation band is applied to the anterior chest wall [8]. This reduces signal from the soft tissues in the chest wall and more clearly distinguishes the anterior pericardium from the right ventricular (RV) free wall. Spin-echo sequences are useful in the assessment of the contents of a pericardial effusion. A simple effusion is of low signal on $T_{1}$ weighted images and uniform high signal on $T_{2}$ and STIR (short tau inversion recovery) images. Complex and septated effusions can be readily identified by CMR more accurately than with CT. The signal of these complex effusions is variable, with haemorrhagic effusions often showing increased signal on $T_{1}$ imaging (Figure 2).

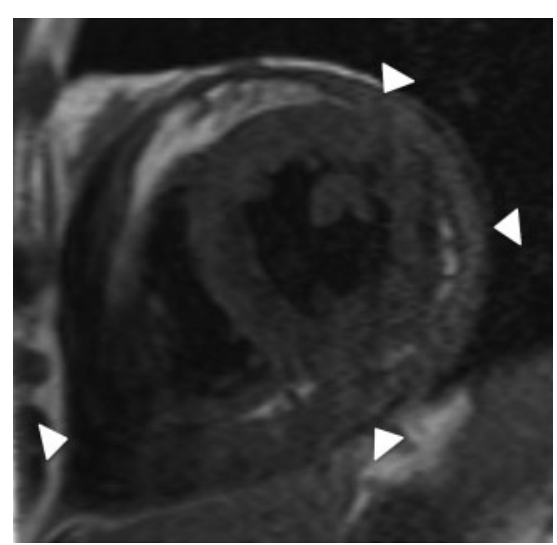

(a)

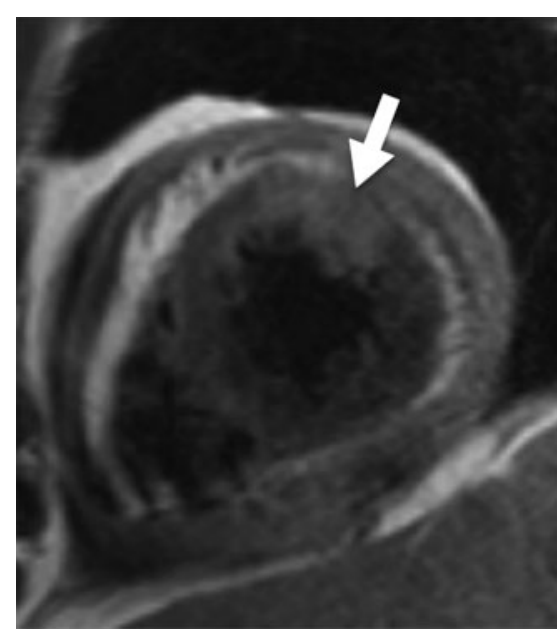

(b)

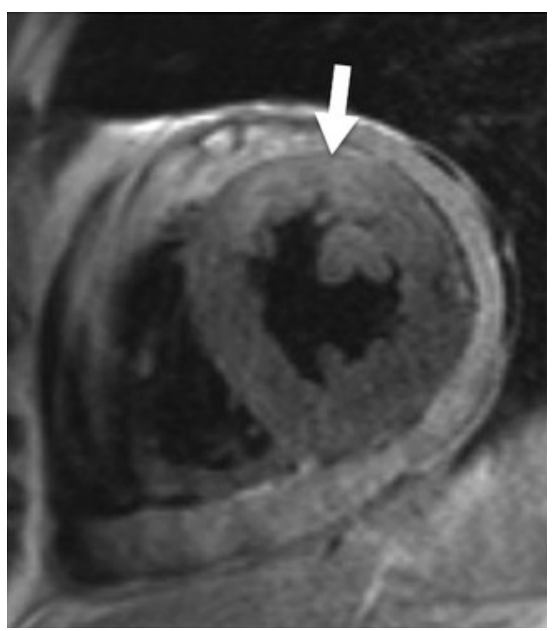

(c)

Figure 2. Short axis spin-echo sequences of a haemorrhagic pericardial effusion (the same case as is shown in Figure 1). (a) $T_{1}$ weighted, (b) $T_{2}$ weighted and (c) short tau inversion recovery (STIR) images demonstrate the effusion, showing mixed areas of low and high signal that indicate its complexity (arrowheads). The $T_{2}$ weighted and STIR images demonstrate an area of high signal in the anterolateral wall (arrows), indicating oedema secondary to a recent myocardial infarct. 


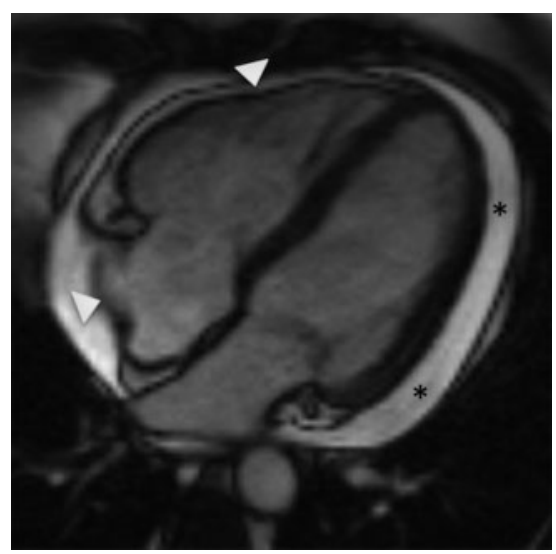

(a)

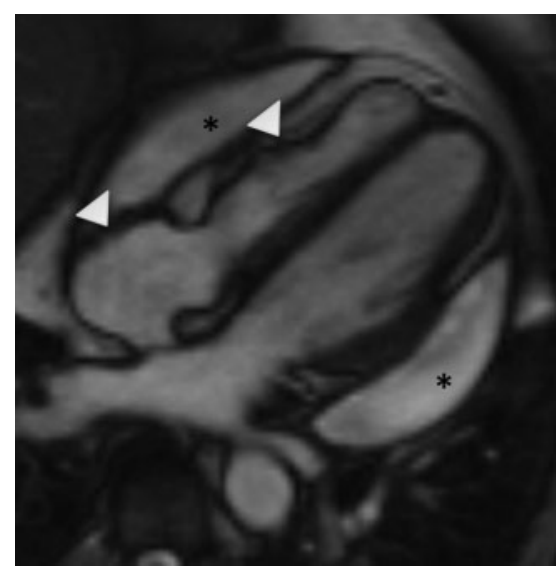

(b)
Figure 3. (a) A simple pericardial effusion on a diastolic-phase steadystate free precession cine image. The right ventricular and right atrial free walls (arrowheads) have a normal configuration without flattening or distortion, suggesting normal diastolic filling without constriction. (b) A diastolic phase cine image of effusive-constrictive pericarditis. The pericardial effusion $(*)$ is causing flattening of the right ventricle and right atrium (arrowheads) with a tubular ventricular morphology.

\section{Steady-state free precession gradient echo cine sequences}

Functional imaging is important in pericardial disease, particularly in pericardial constriction. Pericardial mobility can be assessed with high spatial and temporal resolution, but it is the effect of the pericardium on ventricular contraction that is most important. The ventricles can become distorted, and while systolic function may be maintained, diastolic function is usually significantly impaired (Figure 3).

\section{Spatial modulation of magnetisation cine images}

Tagging sequences involve the superimposition of gridlines on the cardiac images at end diastole. The degree of distortion of these gridlines throughout the cardiac cycle gives a detailed assessment of regional contractility. In pericardial constriction, the pericardium is seen as a rigid, non-compliant structure with adhesions between the pericardial layers and the adjacent myocardium, which impair contractile function $[9,10]$ (Figure 4).

\section{Real-time cine imaging during dynamic respiration}

Real-time sequences provide rapidly acquired, relatively low-resolution cine images. These can be performed during free respiration, and hence the effects of respiration on cardiac function can be determined. This is particularly important in patients with pericardial constriction, where ventricular coupling results in a dramatic flattening of the interventricular septum in early diastole during inspiration (see section on constrictive pericarditis) [11, 12].

\section{Phase-contrast flow imaging}

Velocity-encoded CMR allows accurate assessment of flow across the cardiac valves. Diastolic filling of the ventricles is abnormal in both restrictive cardiomyopathy and pericardial constriction, and this is reflected in mitral and tricuspid flow patterns. Phase-contrast imaging may help to differentiate the underlying physiology [13].

\section{Pericardial anatomy and function}

The pericardium is a fibrous sac that surrounds the heart. It consists of two layers: the visceral pericardium and the parietal pericardium. The visceral pericardium is an inner serosal membrane adherent to the cardiac epicardium. The parietal pericardium consists of an outer fibrocollagenous layer and an inner serosal layer, which is a reflection of the visceral pericardium. There is a potential space between the visceral and parietal pericardium, which can normally contain $15-35 \mathrm{ml}$ of serous fluid distributed mostly over the atrioventricular (AV) and interventricular grooves [14]. This trace of fluid is often seen, particularly within the pericardial recesses, on both CT and CMR.

At the reflections of the pericardium between the great vessels at the base of the heart, the pericardial cavity forms

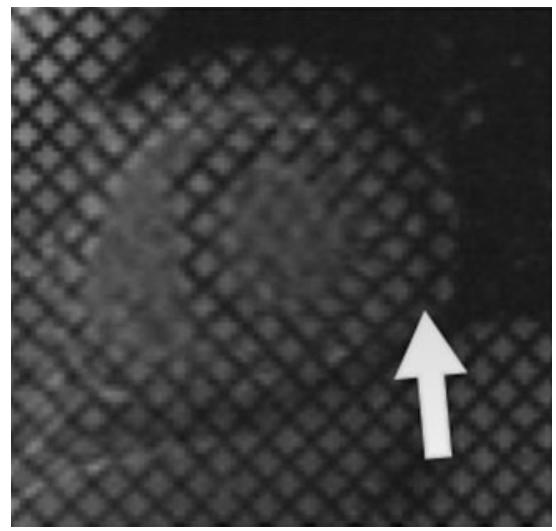

(a)

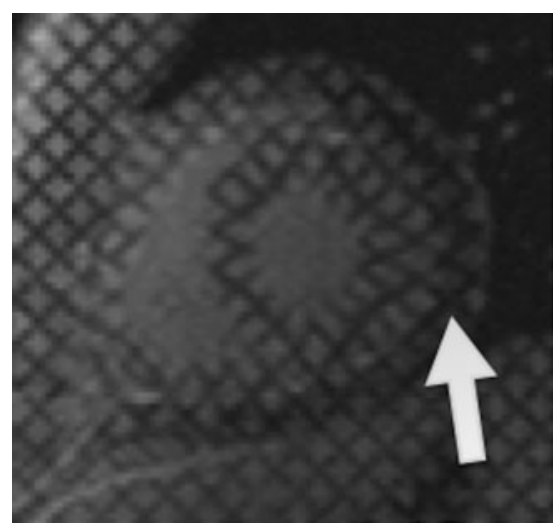

(b)
Figure 4. (a) Diastolic and (b) systolic tagged images in a case of surgically proven constrictive pericarditis. Although the tag lines over the myocardium of the lateral wall distort normally between diastole and systole, they remain intact at the pericardial-myocardial interface (arrows), indicating abnormal pericardial adhesions. 


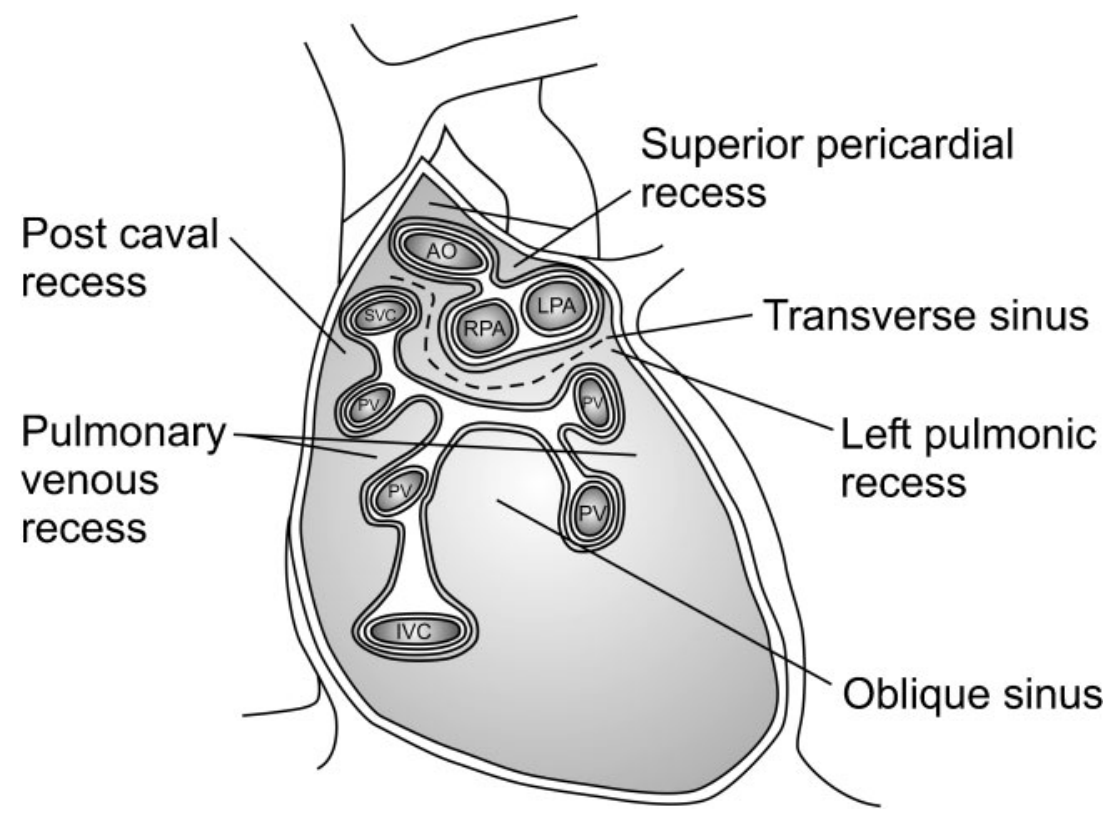

Figure 5. Line diagram demonstrating the posterior pericardial reflections, which form the transverse and oblique sinuses and their associated recesses.

the pericardial sinuses. These are not separate compartments but extensions of the pericardial cavity. The pericardium contains two major pericardial sinuses, transverse and oblique, which communicate with the different recesses [15] (Figure 5). An understanding of the anatomy of these recesses is important in interpreting cross-sectional imaging so as not to confuse them with pathology.

The transverse sinus is a communication between two rolls of pericardium, one surrounding the aorta and pulmonary artery, the other the vena cavae and pulmonary veins. The superior pericardial recess arises from the transverse sinus and is frequently seen adjacent to the ascending aorta on axial cross-sections. This must

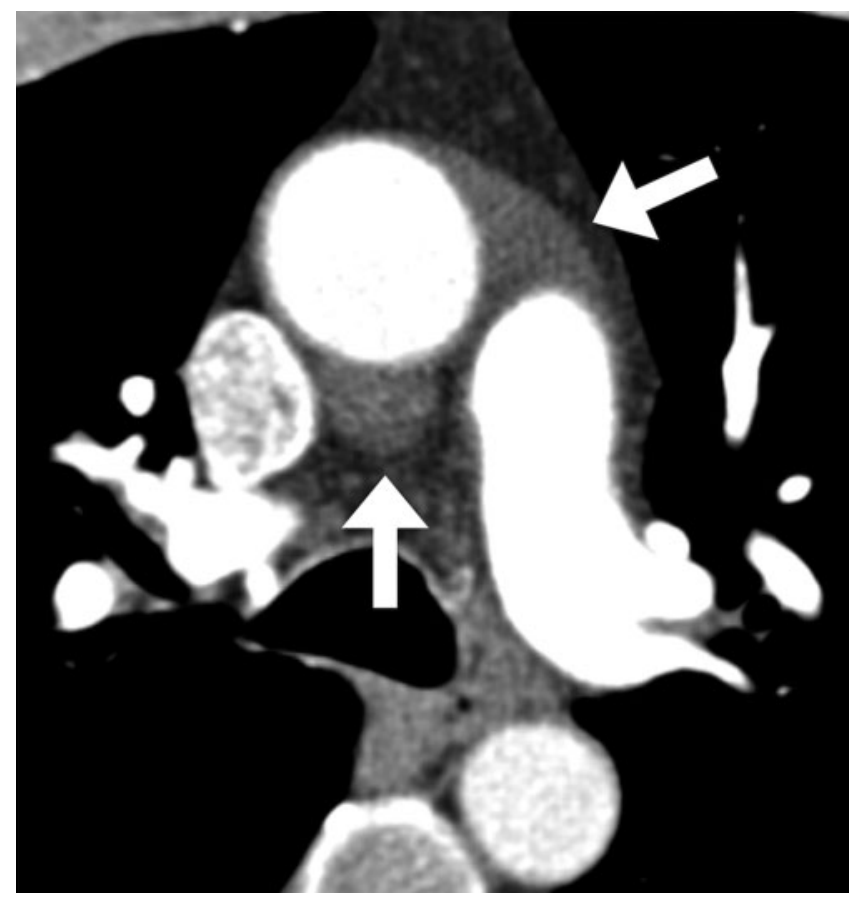

Figure 6. CT of the superior mediastinum demonstrating the superior pericardial recesses (arrows) lateral and posterior to the ascending aorta. not be confused for mediastinal adenopathy or an abnormality of the aortic wall (Figure 6).

The oblique sinus lies behind the left atrium, inferior to the transverse sinus and separated by pericardial reflections, so that part of the posterior wall of the atrium is actually separated from the pericardial space [8] (Figure 7).

The thickness of the normal pericardium as measured on CT and MR is less than $2 \mathrm{~mm}[16,17]$. Anatomical studies have shown the true pericardium to be thinner than this $(0.4-1 \mathrm{~mm})$; the reasons for this discrepancy are thought to be motion artefact and the limits of spatial resolution. Chemical-shift artefact may also occur on CMR at the pericardial fat-fluid interface if a small effusion is present, causing further apparent pericardial thickening [8]. It is for these last two reasons, spatial resolution and chemical-shift artefact, that pericardium can appear thicker on gradient steady-state free precession (SSFP) cine imaging than on single-slice black-blood images (spin-echo sequences).

On CT, the pericardium is identified as a higher attenuation linear structure between the low density epicardial and mediastinal fat. On MR, it is of low signal on $T_{1}$ and $T_{2}$ weighted images, and again is easily seen when adjacent to high signal fat. It is for this reason that the pericardium is best appreciated over the right atrium and right ventricle. It is often not visible over the lateral wall of the left ventricle because of the paucity of fat here (Figure 8).

The pericardium attaches to the sternum, diaphragm and anterior mediastinum, anchoring the heart in the centre of the thorax. It may appear a little thicker at these insertions. Because of its location, it has been suggested that the pericardium is a barrier to infection [14]. The relatively inelastic nature of the pericardium means that it limits acute cardiac dilatation and enhances the mechanical interactions of the heart chambers [18]. In the presence of longstanding stress, however, the pericardium can dilate, thus allowing chronic pericardial effusions to develop without significant impact on cardiac function. The physiological consequences of abnormal pericardial function are discussed in detail in the section on pericardial constriction. 


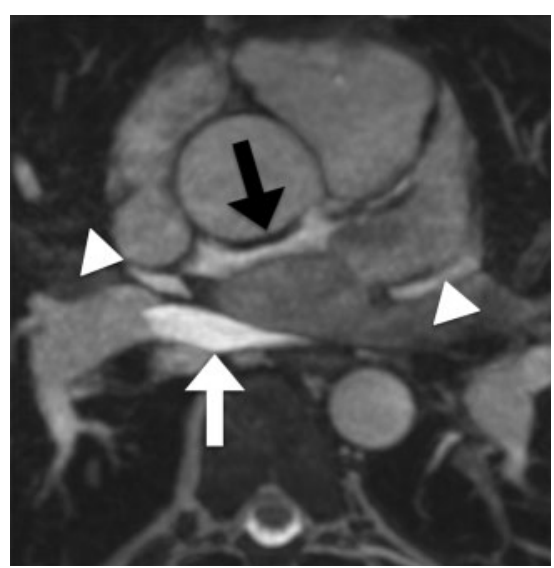

(a)

\section{Congenital pericardial anomalies}

\section{Pericardial cyst}

Pericardial cysts are congenital encapsulated cysts that arise from the pericardium in early development but do not communicate with the pericardial cavity. They are rare, with an incidence of 1 in 100000, but remain the most common benign pericardial mass [19]. They can occur anywhere in the mediastinum, but are most common at the right cardiophrenic angle [4]. They are usually welldefined, round or elliptical lesions of variable size, with thin walls and no internal septation. On CT, they are usually of the same density as water but may show higher attenuation if complicated by haemorrhage or infection (Figure 9). On MRI, pericardial cysts typically have low or intermediate signal intensity on $T_{1}$ weighted images and uniform high signal on $T_{2}$ weighted imaging. Occasionally, high signal is seen on $T_{1}$ weighted imaging, indicating the presence of highly proteinaceous fluid. Pericardial cysts do not exhibit contrast enhancement (Figure 10). Typically, the lesions are found incidentally and are asymptomatic, but occasionally they become symptomatic. This can be secondary to compression of other cardiac structures, infection or cyst rupture $[8,20,21]$.

\section{Pericardial diverticulum}

A pericardial diverticulum can look identical to a pericardial cyst but corresponds to a herniation through a defect in the pericardium that is in contact with the pericardial cavity. This entity should be suspected when
Figure 7. Electrocardiogram ECG and respiratory-gated (navigator) gradient echo (a) axial and (b) sagittal images. This sequence clearly demonstrates the posterior pericardial reflections in a patient with a small pericardial effusion. The transverse sinus (black arrow) lies posterior to the proximal ascending aorta, and this gives rise to the superior pericardial recess more superiorly (asterisk). The oblique sinus is posterior to the left atrium (white arrow). Laterally, the left and right pulmonic recesses (arrowheads) can be seen.

(b)

a complete wall cannot be identified in all parts of the lesion [22]. A pericardial diverticulum can be congenital or acquired, and can result from a failure in the fusion of one of the mesenchymal lacunae that normally combine to form the pericardial sac [8].

\section{Pericardial defect}

Congenital absence of the pericardium is a rare anomaly. It encompasses a range of congenital pericardial defects from a small foramen in the pericardium to a complete absence of the entire pericardium [23]. In most cases, these defects are on the left side of the heart, but they can be located anywhere in the pericardium. Pericardial defects may be associated with other congenital cardiac abnormalities such as atrial septal defect (ASD), patent ductus arteriosus (PDA), bicuspid aortic valve or pulmonary malformations [24].

As previously described, it can be difficult to visualise the normal pericardium over the left side of the heart. As a consequence, cross-sectional imaging is largely reliant on identifying indirect signs of an absent pericardium. These include excessive laevorotation of the heart, interposition of lung tissue between the aorta and main pulmonary artery or between the inferior surface of the heart and diaphragm, and (in partial absence) cardiac indentation at the location of the defect [25-27]. CMR provides further benefit in assessing indirect findings of pericardial absence in its ability to assess function. Physiologically, the left ventricular (LV) apex is normally relatively stationary during the cardiac cycle, but

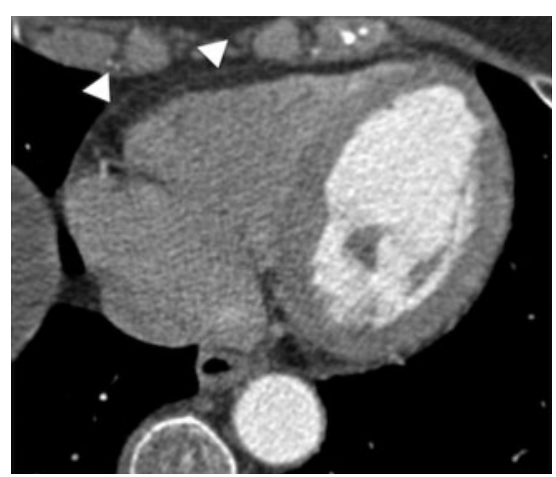

(a)

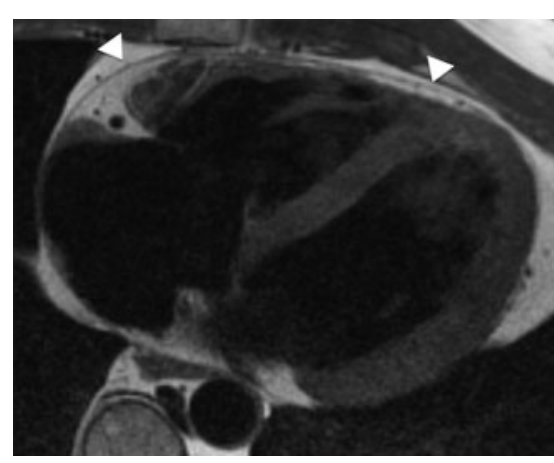

(b)
Figure 8. (a) Axial CT and (b) $T_{1}$ black-blood cardiac magnetic resonance images demonstrating normal pericardium, which is best appreciated over the right ventricle and right atrioventricular groove (arrowheads). 


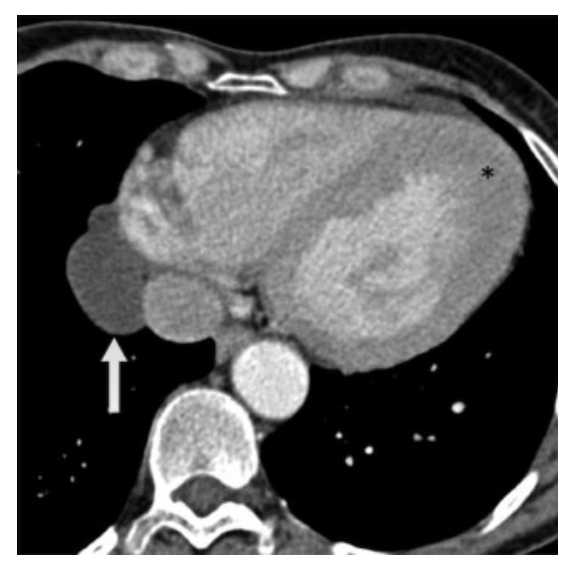

(a)

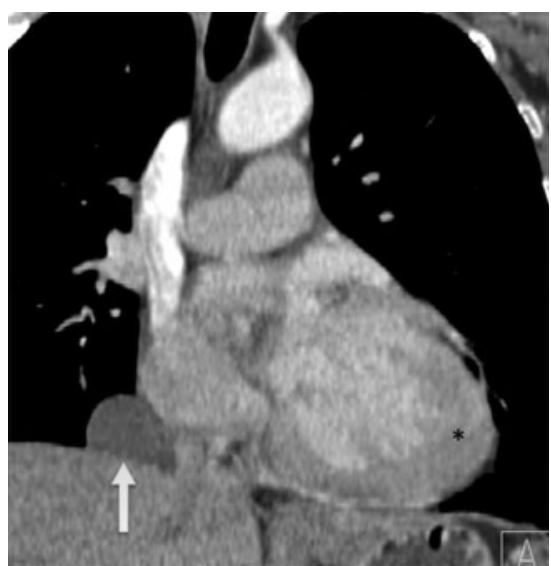

(b)
Figure 9. (a) Axial and (b) coronal CT images of a pericardial cyst (arrow) with the same density as water lying in a typical position adjacent to the pericardium at the right cardiophrenic angle. Incidental note is made of apical left ventricular myocardial hypertrophy $(*)$; this was confirmed on cardiac MRI as apical hypertrophic cardiomyopathy. without the confines of the pericardium, excessive apical motion is demonstrated on cine CMR [28]. Complete absence of the pericardium is considered to be benign, but partial defects do cause complications such as herniation of cardiac structures. Left atrial (LA) appendage entrapment can cause ischaemic necrosis, and compression of the left coronary artery secondary to herniation may cause myocardial ischaemia. In these cases, surgical correction should be considered to relieve the herniation.

\section{Acquired pericardial disease}

\section{Pericarditis}

Inflammation of the pericardium is caused by a wide range of pathologies (Table 1) [14, 29]. The result is thickening of the pericardium with or without a pericardial effusion. Pericardial thickening of greater than $4 \mathrm{~mm}$ on CT is considered abnormal [5], but the thickening may be patchy, and consequently imaging of the entire pericardium is needed $[30,31]$. The role of CT and MRI is to accurately identify pericardial thickening, with or without effusion, which, in the appropriate clinical context, will confirm the diagnosis. Both techniques can demonstrate pericardial enhancement during active inflammation, but MRI provides a more confident visualisation of associated pericardial fluid that may be interpreted as thickening alone on CT [4, 17, 32] (Figure 11). MRI also has the potential to identify acute myocardial inflammation, which may be a diagnostic clue in cases of myopericarditis or occult myocardial infarct (Dressler's syndrome) (see Figure 2). The acute inflammatory phase of pericarditis may resolve completely or follow a more chronic course, with the potential to result in pericardial constriction. Compromise of ventricular function can occur at any stage of this process, either secondary to the presence of a large or rapidly accumulating effusion (tamponade) or owing to pericardial thickening (constriction).

\section{Pericardial effusion}

A small volume $(15-35 \mathrm{ml})$ of pericardial fluid is normal and is frequently seen in the pericardial reflections on CT and MRI. There are no specific criteria for the differentiation of physiological fluid from a pathological effusion, but a depth of fluid of greater than $5 \mathrm{~mm}$ anterior to the right ventricle is considered a moderate effusion (100-500 ml) on MRI [33]. Given the large field of view of CT and MRI (compared with that of echocardiography), these modalities both play a role in identifying the extent and location of pericardial effusions. CT and MRI also allow an assessment of the physiological consequences of the effusion and may provide clues to the aetiology.

The causes of pericardial effusion can be broadly divided into exudates and transudates. Exudates tend to be associated with inflammatory aetiologies of

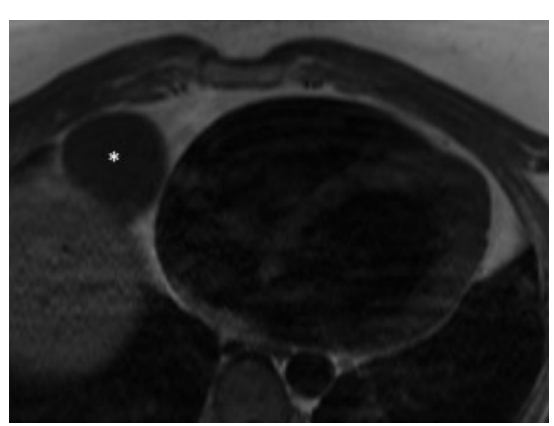

(a)

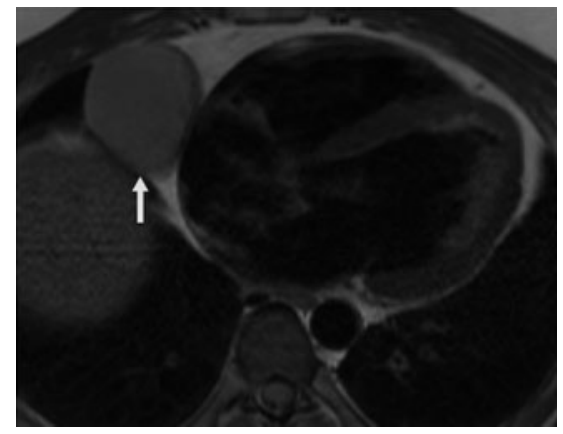

(b)

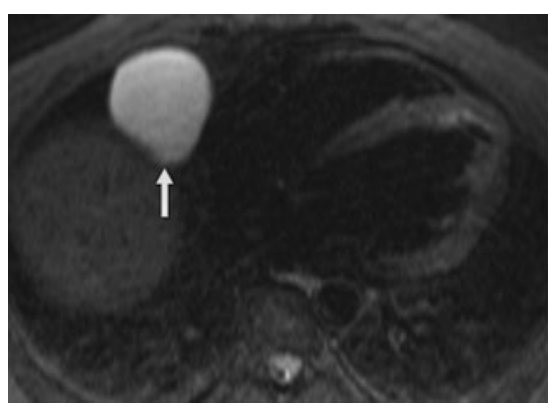

(c)

Figure 10. Axial MRI spin-echo images of a typical pericardial cyst. (a) A $T_{1}$ weighted image shows low signal in the cyst (*). (b) $T_{2}$ weighted and (c) short tau inversion recovery images demonstrate high signal that is suggestive of fluid content (arrows). 
Table 1. Causes of pericarditis

\author{
Idiopathic \\ Infectious (viral, tuberculous, bacterial or fungal) \\ Radiotherapy \\ Neoplasm \\ Autoimmune (systemic lupus erythematosus, rheumatoid arthritis, systemic sclerosis or ankylosing spondylitis) \\ Uraemia \\ Cardiac injury (surgery or trauma) \\ Myocardial infarction [acute or chronic (Dressler's syndrome)]
}

effusion, such as those listed in Table 1. Transudates are typical with heart failure and hypoproteinaemic states. Haemorrhagic effusions are usually associated with acute cardiac or aortic injury, such as trauma, aortic dissection or cardiac rupture. Haemorrhage may, however, complicate any other cause of pericardial effusion. Chylous pericardial effusions are uncommon and are generally related to damage or obstruction to lymphatic drainage secondary to trauma (including surgery) and malignancy.

The primary role for cross-sectional imaging is to determine an underlying cause of the effusion if this is unknown. Exclusion of thoracic malignancy, such as lymphoma, mesothelioma or bronchogenic carcinoma, is best done with CT given its wide field of view and excellent spatial resolution, although MRI also has a role. Specifically, MRI using both SSFP cine and spin-echo sequences is useful in identifying pericardial invasion in patients with known thoracic malignancy, such as bronchogenic or thymic malignancy. In the absence of an obvious cause for an effusion, CT and MRI may provide useful indicators as to the nature of the effusion and its likely aetiology. The imaging characteristics of the fluid on MRI and its attenuation on CT can theoretically be used to differentiate exudates, transudates, haemorrhage and chyle, although in practice this is often not possible. On CT, acute haemorrhage is high density tissue (i.e. density greater than that of skeletal muscle or myocardium) and effusions that are related to exudates, malignancy or hypothyroidism and have higher attenuation than water [6]. On MRI, simple transudates are generally low signal on $T_{1}$ weighted spin-echo sequences and high signal on $T_{2}$ weighted spin-echo sequences. Exudates have high cellular and protein content and are usually of intermediate signal on both $T_{1}$ and $T_{2}$ weighted spin-echo imaging. Flow-related artefacts often cause areas of mixed signal within the effusion on spinecho sequences, making assessment difficult. Gradient echo cine sequences are often more reliable for demonstrating effusion complexity as they may show septations and areas of clot [8].

The appearance of the pericardium itself on MRI or CT is usually more useful in determining the cause of the effusion than the characteristics of the fluid. Thickened pericardium suggests an exudative cause, whereas a thin pericardium is indicative of a transudate. Nodular or irregular pericardial thickening raises the possibility of a neoplastic cause, but note that other inflammatory processes [such as tuberculosis (TB)] may also result in irregular pericardial thickening (Figure 12).

\section{Constrictive pericarditis}

Constrictive pericardial (CP) disease is the end result of a number of disease processes that cause pericardial inflammation (Table 1). Tuberculous pericarditis traditionally accounted for most cases, but post-viral pericarditis, cardiac surgery, thoracic radiotherapy and idiopathic diseases are now more common causes [34, 35]. Pathologically, there is thickening and fibrosis of the visceral and parietal pericardium, often with adhesions to the adjacent myocardium. About $50 \%$ of patients show some pericardial calcification [36].

The main physiological consequences of pericardial constriction are fixed intrapericardial volume and impaired myocardial filling [37]. The symptoms and signs of $\mathrm{CP}$ are therefore predominantly those of rightsided heart failure, and the treatment is to strip the pericardium surgically. It is essential to differentiate $\mathrm{CP}$

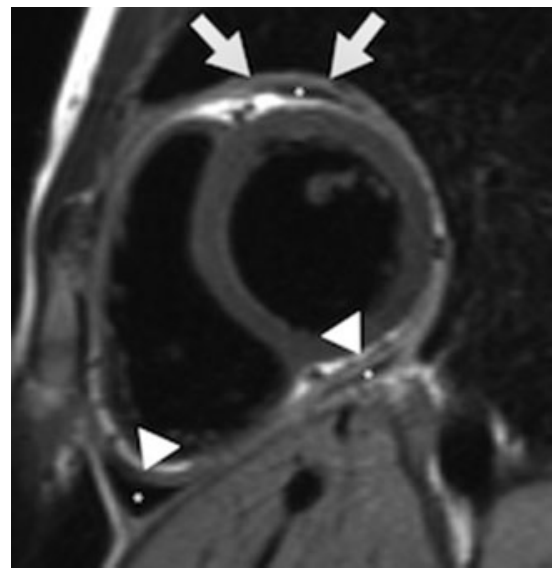

(a)

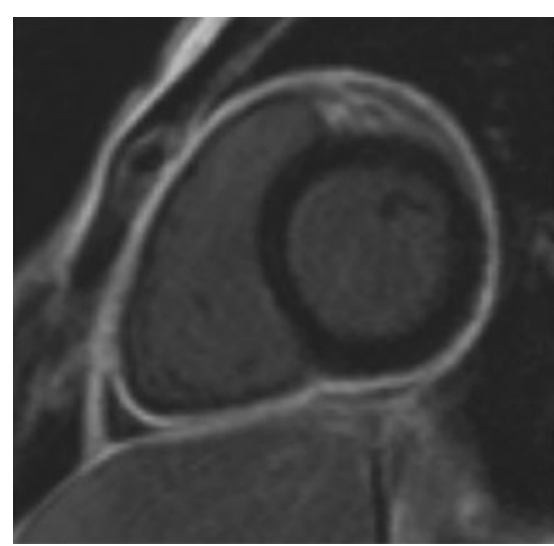

(b)
Figure 11. (a) Short-axis $T_{1}$ weighted black-blood image of a patient with idiopathic pericarditis. Visceral (arrowheads) and parietal (arrows) pericardial thickening are separated by a small pericardial effusion (*). (b) Short-axis delayed enhanced image showing enhancement of the pericardial layers. 


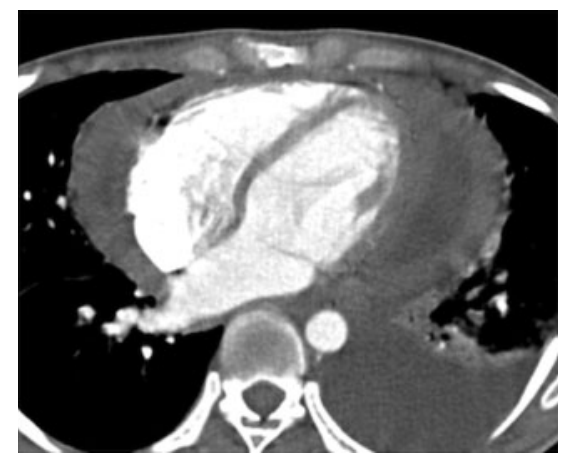

(a)

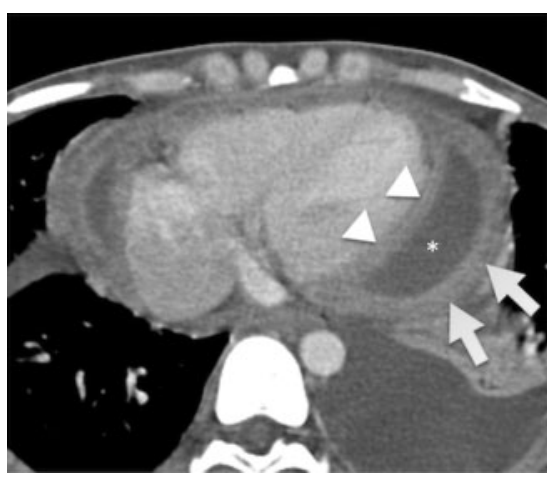

(b)
Figure 12. Axial CT images of a patient with tuberculosis pericarditis. (a) The arterial phase image allows assessment of the ventricular chambers and the position of the interventricular septum. (b) The venous phase image gives better visualisation of the visceral (arrowheads) and parietal (arrows) pericardial thickening and enhancement, with the pericardial effusion separating them $(*)$. The thickness and enhancement of the pericardial layers, rather than the attenuation of the effusion, suggests an exudate rather than a transudate. from other conditions that present with right-sided heart failure, specifically restrictive cardiomyopathy, which has a very similar physiology but completely different treatment. The diagnosis of $\mathrm{CP}$ generally requires both the demonstration of an appropriate physiology and evidence of pericardial thickening. The role of crosssectional imaging is therefore primarily to identify pericardial thickening in the context of a patient with suggestive constrictive physiology on echocardiography and/or catheter angiography. CT and MRI do, however, provide additional information indicative of a constrictive physiology, which will be discussed below. Rightand left-heart catheterisation allows direct measurement of RV and LV filling pressures. Synchronous measurement of RV and LV pressures shows equalisation of diastolic filling pressures (usually to within $3-5 \mathrm{mmHg}$ ) and a typical dip-and-plateau waveform. In subclinical cases, these findings may be unmasked by infusion of a bolus of saline during the study [24].

Pericardial thickening on MRI of greater than $4 \mathrm{~mm}$ differentiates CP from restrictive cardiomyopathy with a sensitivity and specificity of $88 \%$ and $100 \%$, respectively [38] (Figure 13). CT has the advantage of detecting tiny amounts of calcification, although the literature provides no evidence on the sensitivity and specificity of CT-defined pericardial calcification in the diagnosis of CP. Pericardial thickening is often patchy, being most easily appreciated over the RV free wall and AV groove, where it is outlined by pericardial fat [38] (Figure 14). As discussed above, patchy pericardial thickening alone, in the absence of appropriate physiology, should not lead to the diagnosis of pericardial constriction.

Morphological features that indirectly suggest a constrictive physiology may be present on CT and MRI. Evidence of high ventricular filling pressures, such as dilatation of the superior and inferior vena cavae, hepatic veins, coronary sinus and atria, are seen in both $C P$ and restrictive cardiomyopathy. Ascites and pleural effusions are common and again simply reflect high atrial pressures. More specific to pericardial constriction is distortion of the ventricular chambers such that they have a tubular morphology, particularly around the AV groove (Figure 14). Flattening of the RV free wall is also typical (Figure 3) [38, 39]. MRI using tagged images may show evidence of pericardial adhesions to the myocardium, helping to identify functionally important pericardial thickening (Figure 4) [9, 10].

MRI also provides direct functional evidence of $\mathrm{CP}$ that aids its differentiation from restrictive cardiomyopathy. To fully appreciate these findings, a more detailed understanding of the physiology of $\mathrm{CP}$ is required. In a normal heart, the filling pressures of the ventricles are independent of each other, whereas in constriction, the fixed pericardial volume forces the ventricles to compete for diastolic filling, and hence diastolic ventricular pressures equalise. This abnormal interaction is called ventricular "interdependence" or ventricular "coupling" [40-42]. The result is that the interventricular septal

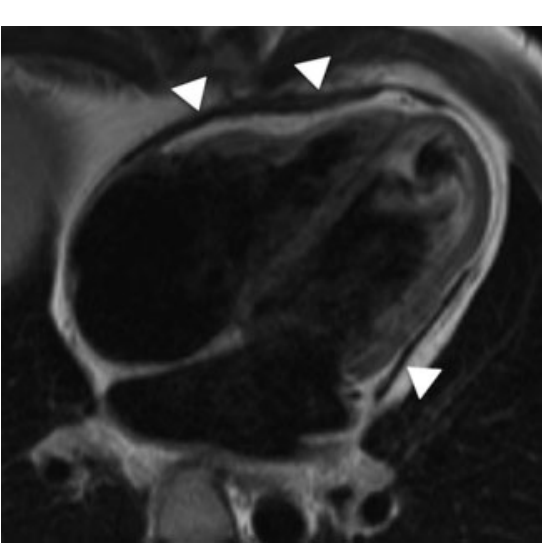

(a)

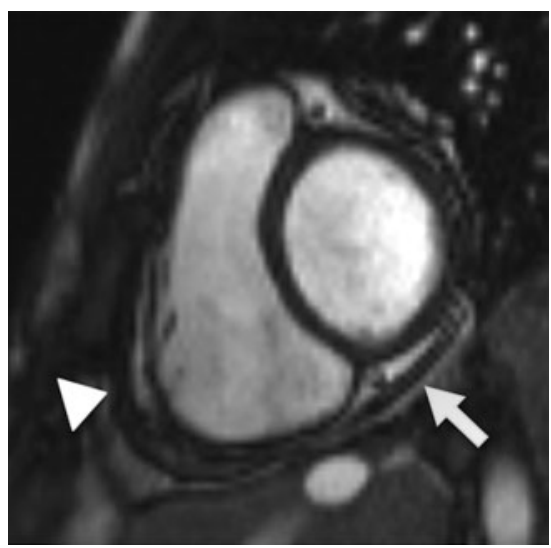

(b)
Figure 13. (a) A black-blood fourchamber MRI showing patchy pericardial thickening of $>4 \mathrm{~mm}$ (arrowheads) in a patient with proven pericardial constriction (Figures 14-17). (b) A still image from a short-axis cine demonstrates the pericardial thickening most clearly over the right ventricular free wall (arrowhead) and inferior interventricular groove (arrow), where the pericardium is surrounded by fat. 


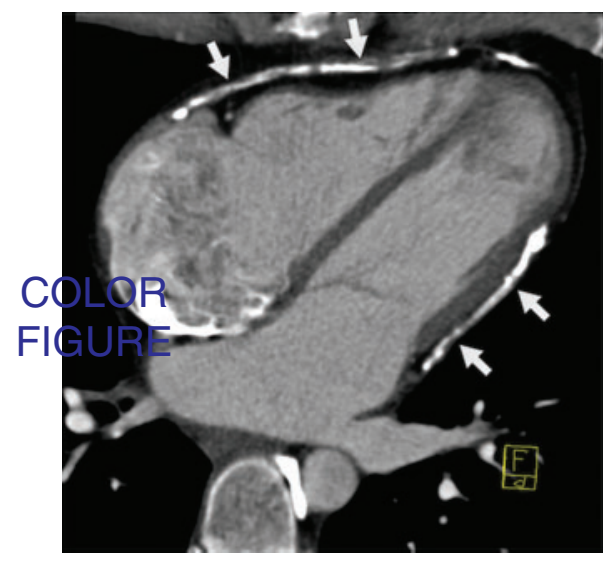

(a)

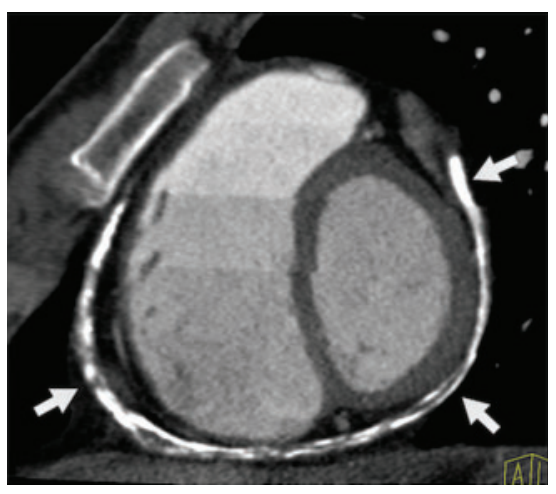

(b)

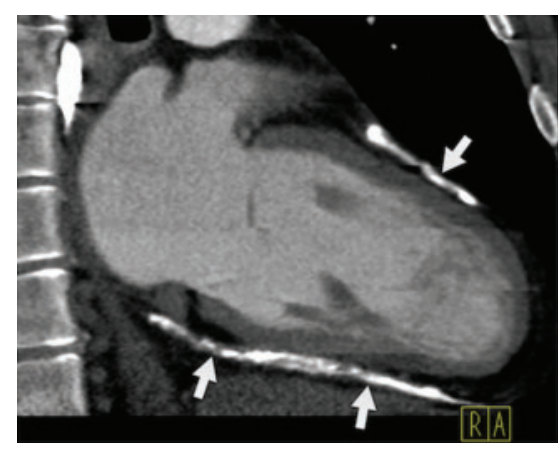

(c)

Figure 14. A prospectively gated CT scan of a patient with constrictive pericarditis reconstructed in (a) four-chamber, (b) shortaxis and (c) two-chamber views. There is extensive calcified pericardial thickening (arrows). The calcification was not appreciated on the MR images (Figure 13). Tubular distortion of the left ventricle and flattening of the right ventricular free wall is indicative of constrictive physiology.

position during diastole will be dependent on the state of filling of the left and right ventricles rather than on any difference in diastolic pressure. This equalisation in pressure accounts for the typical flattening and "bounce" of the interventricular septum in diastole. This effect is exaggerated during inspiration when a negative intrathoracic pressure will cause increased systemic venous return to the right ventricle, which will fill in preference to the left ventricle, causing paradoxical displacement of the septum towards the left ventricle. Conversely, during expiration, pulmonary venous return will be increased and LV filling will be preferred, allowing normalisation of interventricular septal position. This process of respiratory variation in septal position is best demonstrated with a real-time cine MRI sequence made during dynamic respiration, which highlights ventricular septal inversion during early inspiration. This finding differentiates $\mathrm{CP}$ from restrictive cardiomyopathy with a sensitivity of $81 \%$, specificity of $100 \%$ and a positive predictive value of $100 \%$ [11, 41] (Figure 15).

The final features found in $\mathrm{CP}$ relate to the filling pattern of the ventricles. Owing to high atrial pressure,

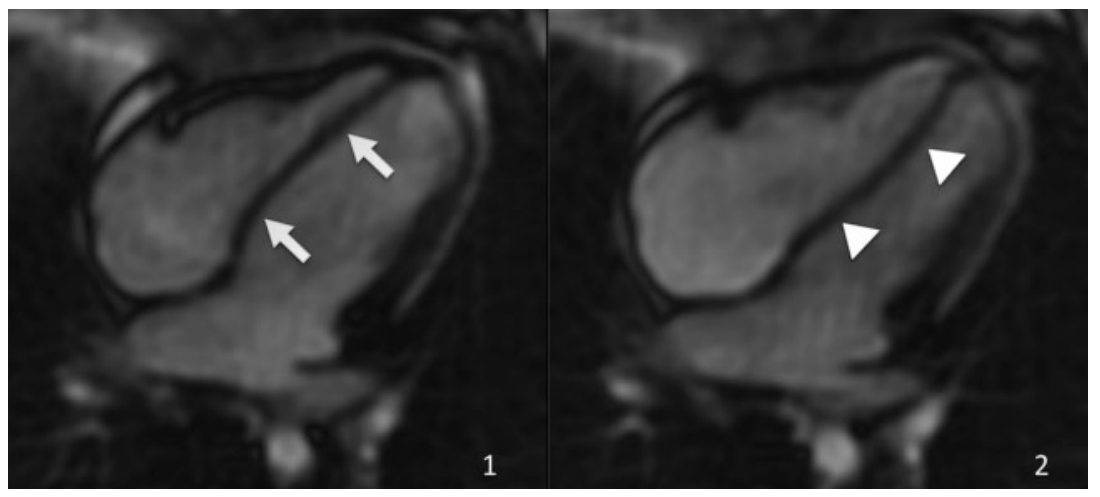

(a)

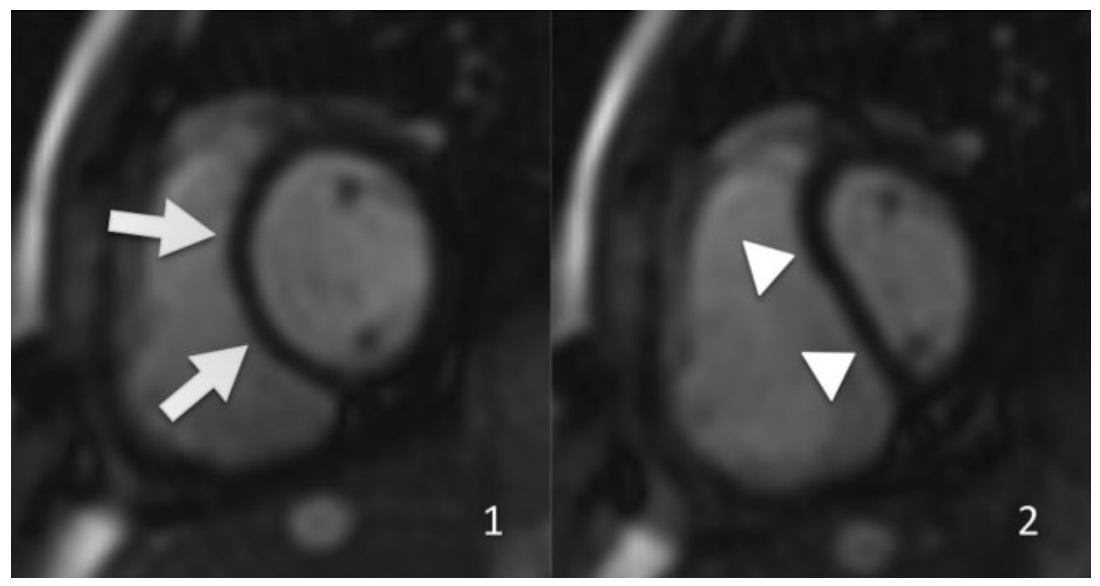

(b)
Figure 15. Real-time cine images of a patient with constrictive pericarditis. (a) Four-chamber and (b) shortaxis views taken in the diastolic phase during (1) expiration and (2) early inspiration. The interventricular septum is normally positioned during expiration (arrows) but inverts during inspiration (arrowheads). 


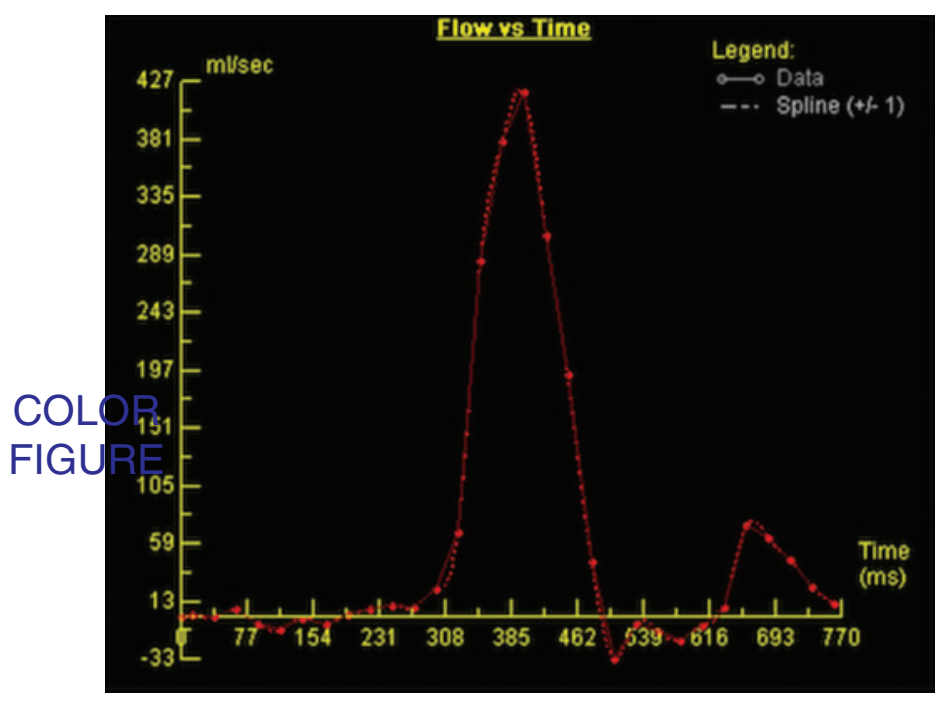

Figure 16. A mitral flow curve from a phase-contrast MRI acquisition shows a dominant $E$ wave and a small $A$ wave with a rapid $\mathrm{E}$ descent, confirming abnormal diastolic filling in a patient with proven constrictive physiology.

the ventricles fill very rapidly in early diastole upon opening of the mitral and tricuspid valves. If present, pericardial constriction then causes an abrupt cessation of ventricular filling. This is manifest on phase-contrast imaging as a dominant $\mathrm{E}$ wave in early diastole and a very small or absent $A$ wave in late diastole (Figure 16). This finding is also seen in advanced restrictive cardiomyopathy. Time-volume curves of LV filling in CP demonstrate the same physiology, with rapid early diastolic filling followed by a plateau phase of reduced filling [13] (Figure 17).

\section{Unusual patterns of constrictive pericarditis}

Unfortunately, there are several presentations of CP that do not have the typical features and cause diagnostic confusion [43].

\section{Constriction with normal pericardial thickness}

Up to $18 \%$ of patients with surgically proven $\mathrm{CP}$ have no evidence of significant pericardial thickening $(<2 \mathrm{~mm})$ [44]. The most common causes of constriction in this group are cardiac surgery, irradiation, previous infarction and idiopathic causes. The important message is that pericardial function rather than thickening alone is the cause of constrictive physiology and that patients with pericardium of normal thickness may benefit from surgery.

\section{Transient pericardial constriction}

Some patients with acute constrictive pericarditis appear to resolve on follow-up echocardiography and clinical assessment. In one series, $16 \%$ of patients showed resolution over 2-3 months [45, 46]. Although most patients with acute pericarditis proceed to complete resolution, there appear to be some who have a transient constrictive physiology, whereas others develop chronic constrictive pericarditis. In more acute presentations, it

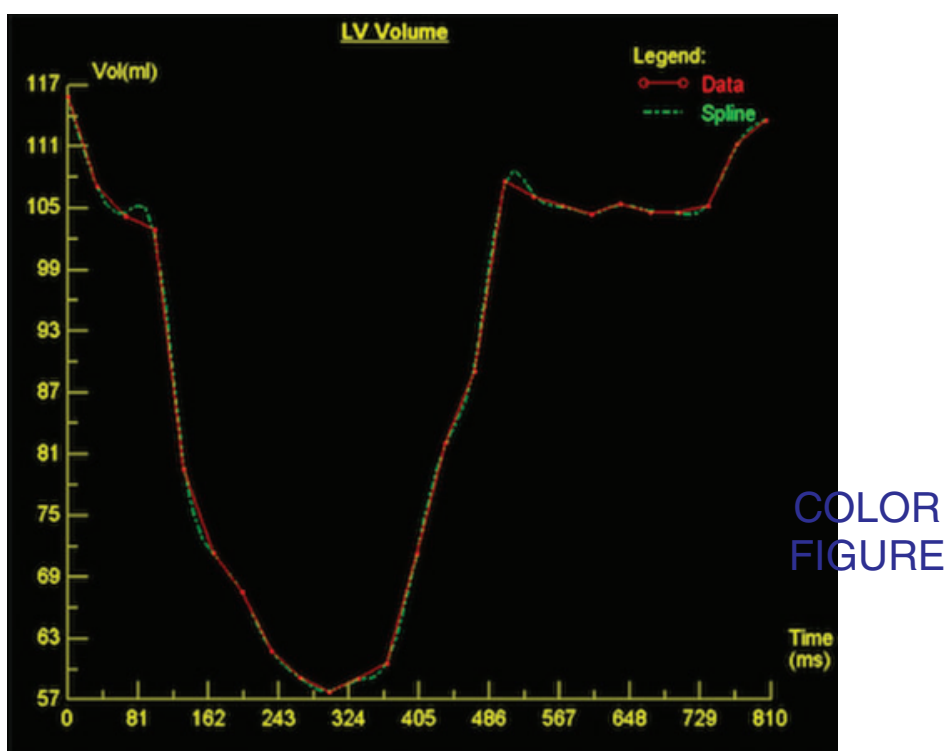

Figure 17. A left ventricular (LV) time-volume curve shows rapid diastolic filling followed by a plateau phase in a patient with proven pericardial constriction (the same case as Figures 13-16).

may therefore be worth pursuing a period of medical therapy before making a decision to offer surgery.

\section{Effusive-constrictive pericarditis}

Effusive-constrictive pericarditis is a syndrome thought to be caused by visceral pericardial constriction. Some patients with pericardial effusion and apparent tamponade at presentation show little or no physiological benefit from pericardiocentesis [47]. Drainage of the effusion reveals the underlying constrictive physiology. Effusive-constrictive pericarditis may resolve or more usually progress to persistent pericardial constriction that requires surgery. It appears to lie on a spectrum of diseases evolving from acute pericarditis to classical CP (Figure 18).

\section{Pericardial tumours}

Tumours of the pericardium are uncommon, and when they do occur, they are more frequently metastases than primary tumours [48]. Pericardial metastases can be difficult to identify on CT and CMR, although there is evidence that $10-12 \%$ of patients dying as a result of malignancy have pericardial metastases at post mortem $[49,50]$. Where these are identified ante mortem, they are associated with poor prognosis. Nodularity with pericardial thickening and enhancement, and occasionally discrete nodules, may be seen on CT or CMR. Pericardial tumours are usually associated with significant pericardial effusion, which tends to be haemorrhagic. The volume of the effusion is usually out of proportion with the amount of tumour visible. The most common primary malignancies that metastasise to the pericardium are lung and breast cancers, although secondary deposits from melanoma and lymphoma are well recognised [50].

Primary tumours of the pericardium are rare. The most common primary malignant tumour is mesothelioma (Figure 19), and this accounts for about $50 \%$ of all 


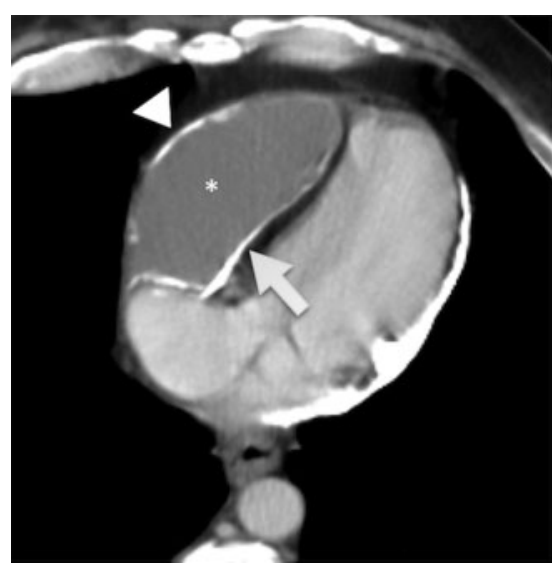

(a)

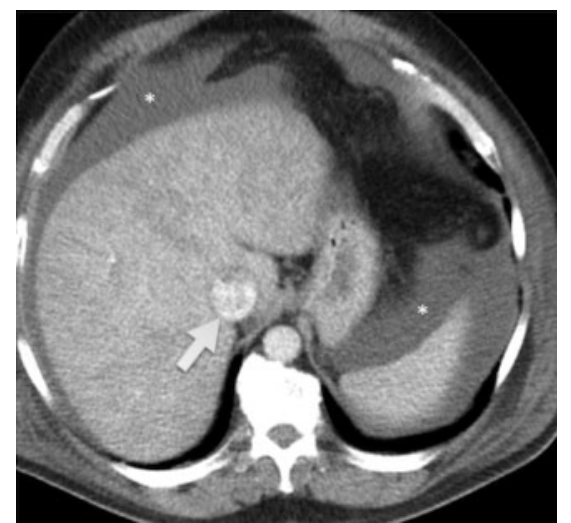

(b)

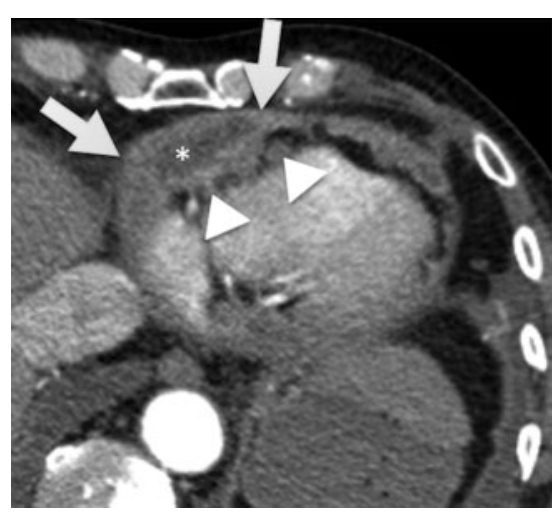

(a)

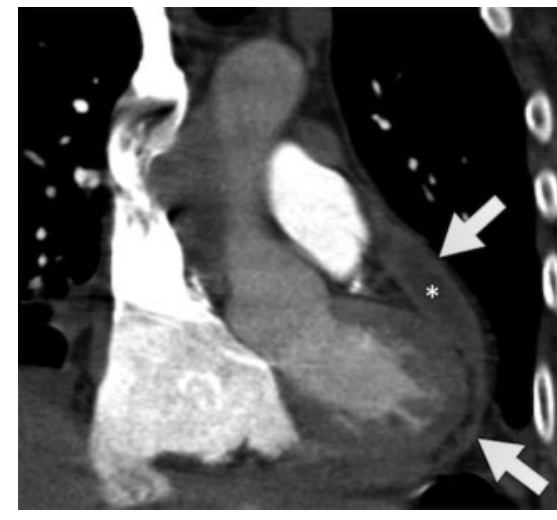

(b)
Figure 18. (a) Axial CT image of a patient with constrictive-effusive pericarditis, demonstrating calcified parietal (arrowhead) and visceral (arrow) pericardium separated by a loculated pericardial effusion $\left(^{*}\right)$. There is marked compression of the right ventricular free wall. (b) Axial image through the upper abdomen shows reflux of contrast into the inferior vena cava (arrow) and ascites $(*)$.

Figure 19. (a) Axial and (b) coronal images from a contrast-enhanced CT of a patient with biopsy-proven pericardial mesothelioma. Irregularly enhanced pericardial thickening (arrows) is invading the epicardial fat (arrowheads) and there is a loculated pericardial effusion (*). The superior vena cava and inferior vena cava (IVC) are dilated and there is reflux of contrast into the IVC, indicating high right ventricular filling pressures and, indirectly, a constrictive physiology.

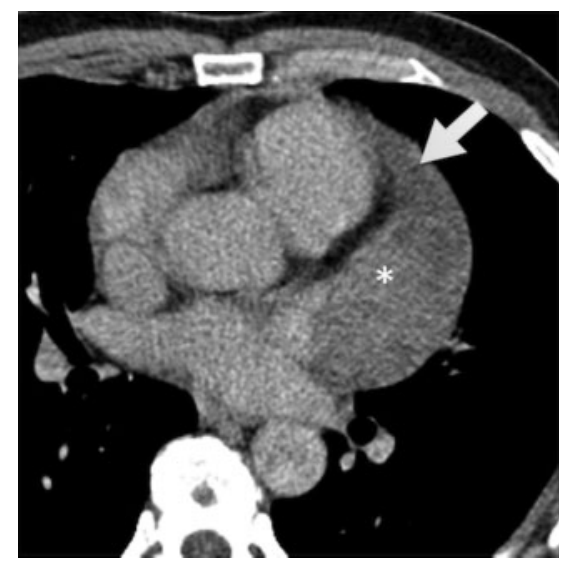

(a)

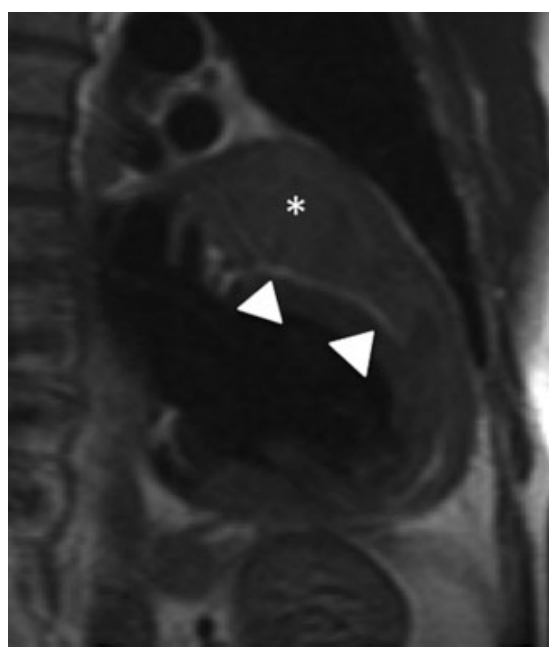

(b)

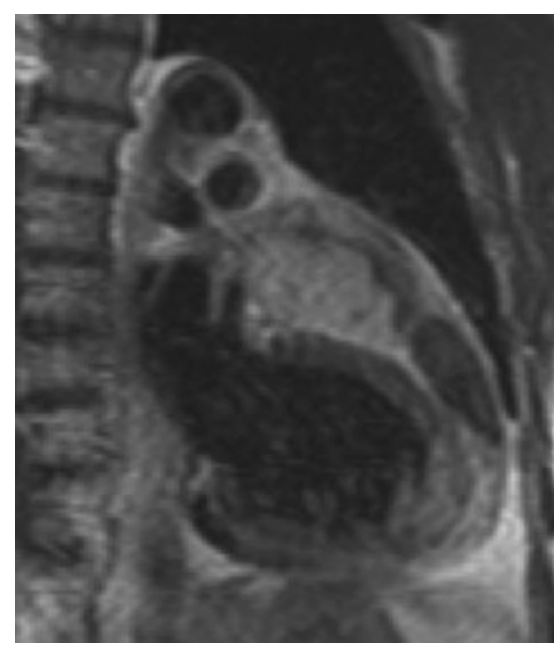

(c)

Figure 20. (a) Axial CT image post contrast of a patient who presented with a pericardial effusion; there is a soft-tissue mass lying in the region of the left atrial appendage $(*)$ and a small pericardial effusion (arrow). (b) A $T_{1}$ weighted spin-echo image confirms a soft-tissue mass $\left({ }^{*}\right)$ arising from the pericardium with a preserved fat plane around the myocardium (arrowheads). (c) Following contrast, the mass enhances, differentiating it from a haematoma. Surgical excision showed a synovial sarcoma arising from the pericardium. 
primary pericardial tumours [51]. Mesothelioma is associated with asbestos exposure, but the incidence of pericardial mesothelioma is less than $1 \%$ of the incidence of pleural mesothelioma. It frequently occurs in middle-aged patients and is more common in males than females. It is typically diffuse, covering most of the pericardial surface, and there is usually a pericardial effusion that is haemorrhagic. Patients may present because of the effects of pericardial constriction, where the thickening of the pericardium is the dominant process, or because of tamponade if there is substantial pericardial effusion. CT and CMR demonstrate the thickened and often irregular pericardium, but it is difficult to distinguish pleural mesothelioma from other causes of pericardial thickening such as TB pericarditis on imaging alone. The imaging features are rather non-specific and a biopsy is usually required for diagnosis.

Other primary malignant pericardial tumours include sarcomas, lymphoma and malignant teratomas [48] (Figure 20). These tend to present as large masses with a blood-stained or frankly haemorrhagic pericardial effusion. Liposarcomas tend to have a high fat content, and thus will be predominantly of low attenuation on CT. On CMR, they demonstrate high signal on $T_{1}$ images and low signal on fat-saturation sequences. In general, the imaging features are non-specific and pericardiocentesis or biopsy is usually required [4].

The most common benign tumour of the pericardium is a lipoma. These focal fatty masses have the typical appearance of fat on CT and CMR imaging and are uniform with no central solid elements, which helps to differentiate them from liposarcomas (Figure 21). Teratomas are also recognised in the pericardium and are more often benign than malignant. These appear as a focal mass containing fat and calcium and, because of the difficulties of demonstrating calcium on MR, these are best imaged with CT. Pericardial fibromas are usually of soft-tissue attenuation on CT, whereas they are seen as low signal masses on $T_{1}$ and $T_{2} \mathrm{MR}$ sequences and can demonstrate significant gadolinium accumulation on late gadolinium enhancement imaging. Gossypibomas are foreign-body granulomas that can occur following trauma or surgery. The appearance of these depends on the nature of the foreign body.

Pericardial haematomas usually occur following trauma or surgery. Their appearance on CT and CMR depends on how long they have been present, but they are usually rather ill defined. On CT, they are seen as a non-enhancing mass of initially increased attenuation, but this attenuation decreases in density with time [36]. Focal calcification can be seen in chronic haematomas. On MR, acute haematomas demonstrate high signal on $T_{1}$, whereas subacute haematomas at 1-4 weeks are heterogeneous with foci of both high and low signal [52]. Chronic haematomas often have a low signal rim on $T_{1}$ imaging with further low signal foci within the lesion, which probably represents calcification.

\section{Conclusion}

Both CT and MRI allow the detailed assessment of the pericardium in both health and disease. Echocardiography remains the initial test of choice, but cross-sectional imaging techniques provide substantial additional information and are frequently used as second-line investigations. The choice of CT or MRI will often depend on local expertise and availability: both reliably identify pericardial thickening and effusion as well as extracardiac causes of pericardial disease. CT has the advantage of speed and spatial resolution with the capability of defining pericardial calcification. MRI involves no ionising radiation and offers benefits in the functional assessment of pericardial disease. The two techniques are therefore complementary and there is often merit in performing both.

A thorough understanding of the appearances of the pericardium and its physiology is a prerequisite for undertaking cross-sectional imaging of the pericardium and for its successful contribution to the diagnosis and management of pericardial disease.

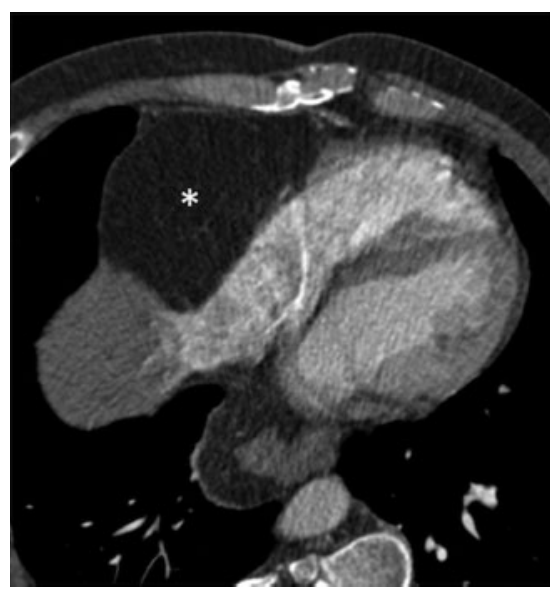

(a)

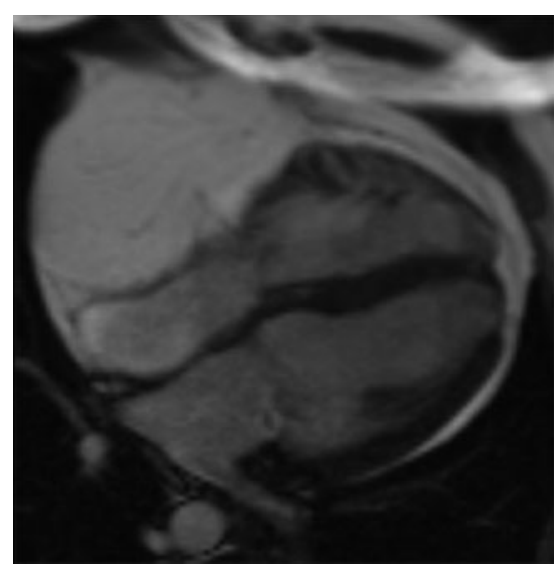

(b)

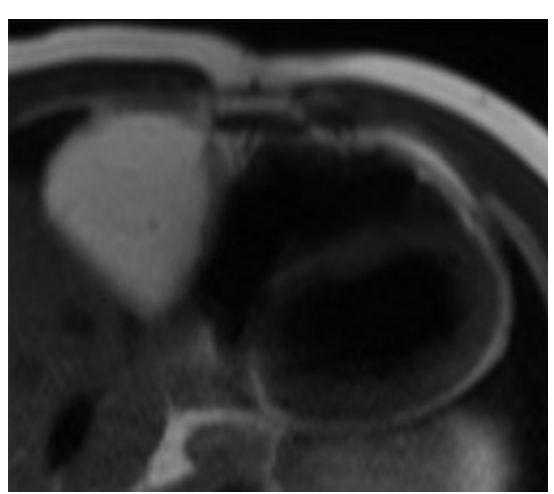

(c)

Figure 21. (a) An axial CT image of a pericardial lipoma (*). The attenuation is identical to that of subcutaneous fat. There is flattening of the right ventricle and right atrium but no direct infiltration. (b) A steady-state free precession cine image and (c) a $T_{1}$ weighted spin-echo image demonstrate high signal within the lipoma, similar to that of subcutaneous fat. The fat signal is homogeneous with no features of a liposarcoma. 


\section{References}

1. Yousem D, Traill TT, Wheeler PS, Fishman EK. Illustrative cases in pericardial effusion misdetection: correlation of echocardiography and CT. Cardiovasc Intervent Radiol 1987;10:162-7.

2. Engel PJ. Echocardiographic findings in pericardial disease In: Fowler NO, editor. The pericardium in health and disease. Armonk, NY: Futura, 1985. pp. 99-151.

3. Palka P, Lange A, Donnelly JE, Nihoyannopoulos P. Differentiation between restrictive cardiomyopathy and constrictive pericarditis by early diastolic Doppler myocardial velocity gradient at the posterior wall. Circulation 2000;102:655-62.

4. Wang Z, Reddy G, Gotway M, Yeh B, Hetts S, Higgins C. $\mathrm{CT}$ and MR imaging of pericardial disease. Radiographics 2003;23:S167-80.

5. Gopalan D, Raj V, Hoey E. Cardiac CT: non-coronary applications. Postgrad Med J 2010;86:165-73.

6. Tomoda H, Hoshiai M, Furuya H, Oeda Y, Matsumoto S, Tanabe $\mathrm{T}$, et al. Evaluation of pericardial effusion with computed tomography. Am Heart J 1980;99:701-6.

7. Misselt AJ, Harris SR, Glockner J, Feng D, Syed IS, Araoz PA. MR imaging of the pericardium. Magn Reson Imaging Clin N Am 2008;16:185-99.

8. Bogaert J, Francone M. Cardiovascular magnetic resonance in pericardial disease. J Cardiovasc Magn Reson 2009;11:14.

9. Kojima S, Yamada N, Goto Y. Diagnosis of constrictive pericarditis by tagged cine magnetic resonance imaging. N Eng J Med 1999;341:373.

10. Mikolich JR, Martin ET. Constrictive pericarditis diagnosed by cardiac magnetic resonance imaging in a pacemaker patient. Circulation 2007;115:e191-3.

11. Francone M, Dymarkowski S, Kalantzi M, Rademakers FE, Bogaert J. Assessment of ventricular coupling with realtime cine MRI and its value to differentiate constrictive pericarditis from restrictive cardiomyopathy. Eur Radiol 2006;16:944-51.

12. Francone M, Dymarkowski S, Verbeken E, Bogaert J. Real time cine MRI of ventricular septal motion. A novel approach to assess ventricular coupling. J Magn Reson Imaging 2005;21: 305-9.

13. Paelinck BP, Lamb HJ, Bax JJ, Van der Wall EE, de Roos A. Assessment of diastolic function by cardiovascular magnetic resonance. Am Heart J 2002;144:198-205.

14. Little WC, Freeman GL. Pericardial disease. Circulation 2006;113:1622-32.

15. Groell R, Schaffler GJ, Rienmueller R. Pericardial sinuses and recesses findings at electrocardiographically triggered electron-beam CT. Radiology 1999;212:69-73.

16. Bull RK, Edwards PD, Dixon AK. CT dimensions of the normal pericardium. Br J Radiol 1998;71:923-5.

17. Sechtem U, Tscholakoff D, Higgins CB. MRI of the abnormal pericardium. AJR Am J Roentgenol 1986;147:245-52.

18. Applegate RJ, Johnstone WE, Vinten-Johansen J, Klopfenstein HS, Little WC. Restraining effect of intact pericardium during acute volume loading. Am J Physiol 1992;262: H1725-HH1733.

19. Hynes JK, Tajik AJ, Osborne MJ, Orszulak TA, Seward JB. Two-dimensional echocardiographic diagnosis of pericardial cyst. Mayo Clin Proc 1983;58:60-3.

20. King JF, Crosby I, Pugh D, Reed W. Rupture of pericardial cyst. Chest 1971;60:611-12.

21. Sharma R, Harden S, Peebles C, Dawkins KD. Percutaneous aspiration of a pericardial cyst: an acceptable treatment for a rare disorder. Heart 2007;93:22.

22. Caretta A, Negri G, Pansera M, Melloni G, Zannini P. Thoracoscopic treatment of a pericardial diverticulum. Surg Endosc 2003;17:158.

23. Nasser WK. Congenital diseases of the pericardium. Cardiovasc Clin 1976;7:271-86.
24. Le Winter M. Pericardial diseases. In: Libby P, Bonow R, Zipes D, Mann D, editors. Braunwald's heart disease. 8th edition. Philadelphia, PA: WB Saunders \& Co., 2008. pp. 1829-53.

25. Abbas AE, Appleton CP, Liu PT, Sweeney JP. Congenital absence of the pericardium: case presentation and review of the literature. Int J Cardiol 2005;98:21-2.

26. Scheuermann-Freestone M, Orchard E, Francis J, Petersen M, Friedrich M, Rashid A, et al. Partial congenital absence of the pericardium. Circulation 2007;116:e126-9.

27. Gatzoulis MA, Munk M, Merchant N, Van Arsdell GS, McCrindle BW, Webb GD. Isolated congenital absence of the pericardium: clinical presentation, diagnosis and management. Ann Thorac Surg 2000;69:1209-15.

28. Psychidis-Papakyritsis P, de Roos A, Kroft LJM. Functional MRI of congenital absence of the pericardium. AJR Am J Roentgenol 2007;189:W312-14.

29. Troughton RW, Asher CR, Klein AL. Pericarditis. Lancet 2004;363:717-27.

30. Breen JF. Imaging of the pericardium. J Thorac Imaging 2001;16:47-54

31. Ben-Horin S, Portnoy O, Pauzner R, Livneh A. Localized pericardial inflammation in systemic lupus erythematosus. Clin Exp Rheumatol 2004;22:483-4.

32. Taylor AM, Dymarkowski S, Verbeken E, Bogaert J. Detection of pericardial inflammation with late-enhancement cardiac magnetic resonance imaging: initial results. Eur Radiol 2006;16:569-74.

33. Frank H, Globits S. Magnetic resonance imaging evaluation of myocardial and pericardial disease. J Magn Reson Imaging 1999;10:617-26.

34. Ling L, Oh J, Schaff H, Danielson G, Mahoney D, Seward J, et al. Constrictive pericarditis in the modern era: evolving clinical spectrum and impact on outcome after pericardiectomy. Circulation 1999;100:1380-6.

35. Cameron J, Oesterle SN, Baldwin JC, Hancock EW. The etiologic spectrum of constrictive pericarditis. Am Heart J 1987;113:354-60.

36. O'Leary SM, Williams PL, Williams MP, Edwards AJ, Roobottom CA, Morgan-Hughes GJ, et al. Imaging the pericardium: appearances on ECG-gated 64-detector row cardiac computed tomography. Br J Radiol 2010;83:194-205.

37. Sengupta PP, Eleid MF, Khandheria BK. Constrictive pericarditis. Circ J 2008;72:1555-62.

38. Masui T, Finck S, Higgins CB. Constrictive pericarditis and restrictive cardiomyopathy: evaluation with MR imaging. Radiology 1992;182:369-73.

39. Oyama N, Komuro K, Nambu T, Manning W, Miyasaka K. Computed tomography and magnetic resonance imaging of the pericardium: anatomy and pathology. Magn Reson Med Sci 2004;3:145-52.

40. Jardin F. Ventricular interdependence: how does it impact on haemodynamic evaluation in clinical practice? Intensive Care Med 2003;29:361-3.

41. Giori B, Mollett NRA, Dymarkowski S, Rademakers FA, Bogaert J. Assessment of ventricular septal motion in patients clinically suspected of constrictive pericarditis, using magnetic resonance imaging. Radiology 2003;228: 417-24.

42. Nishimura RA. Constrictive pericarditis in the modern era: a diagnostic dilemma. Heart 2001;86:619-23.

43. Sagrista-Sauleda J. Pericardial constriction: uncommon patterns. Heart 2004;90:257-8.

44. Talreja DR, Edwards WD, Danielson GK, Schaff HV, Tajik AJ, Tazelaar HD, et al. Constrictive pericarditis in 26 patients with histologically normal pericardial thickness. Circulation 2003;108:1852-7.

45. Sagristà-Sauleda J, Permanyer-Miralda G, Candell-Riera J, Angel J, Soler-Soler J. Transient cardiac constriction: an 
unrecognized pattern of evolution in effusive acute idiopathic pericarditis. Am J Cardiol 1987;59:961-6.

46. Haley JH, Tajik J, Danielson GK, Schaff HV, Mulvagh SL, Oh JK. Transient constrictive pericarditis: causes and natural history. J Am Coll Cardiol 2004;43:271-5.

47. Sagristà-Sauleda J, Angel J, Sanchez A, Permanyer-Miralda G, Soler-Soler J. Effusive-constrictive pericarditis. N Engl J Med 2004;350:469-75.

48. Syed JS, Feng D, Harris SR, Martinez MW, Misselt AJ, Breen $\mathrm{JF}$, et al. MR imaging of cardiac masses. Magn Reson Imaging Clin N Am 2008;16:137-64.
49. Abraham KP, Reddy V, Gattuso P. Neoplasms metastatic to the heart: review of 3314 consecutive autopsies. Am J Cardiovasc Pathol 1990;3:195-8.

50. Klatt EC, Heitz DR. Cardiac metastases. Cancer 1990;65: 1456-9.

51. Grebenc ML, Christenson MLR, Burke AP, Green CE, Galvin JR. Primary cardiac and pericardiac neoplasms: radiologicpathologic correlation. Radiographics 2000;20:1073.

52. Seelos KC, Funari M, Chang JM, Higgins CB. Magnetic resonance imaging in acute and subacute mediastinal bleeding. Am Heart J 1992;123:1269-72. 